\title{
COVID-19 Herd Immunity Simulation with Scratch
}

\author{
Dazhuang Wang ${ }^{1}$ and Leyu Wang ${ }^{2 *}$ \\ ${ }^{1}$ Fairfax County Public Schools, Virginia, USA \\ ${ }^{2}$ Center for Collision Safety and Analysis, George Mason University, USA
}

*Corresponding author: Leyu Wang, Center for Collision Safety and Analysis, George Mason University, USA.

Received Date: February 11, 2021

Published Date: February 26, 2021

\section{Abstract}

The total infected population in the pandemic is found to be related to the number of risky contacts a person may have per day. COVID-19 will infect the entire population if the average person has one risky encounter per day. The virus is unlikely to spread further if the average person has lower than 0.23 times risky encounter per day. In this study, herd immunity for COVID-19 is simulated with scratch. The vaccine was added later as a factor in the study.

\section{Introduction}

Herd immunity is a concept that is frequently mentioned in COVID-19 pandemic. The idea is that the population, which is exposed and immunized to the virus, will serve as a barrier to prevent further spreading of the disease $[1,2]$. It is important to know the percentages of total infected population before herd immunity is achieved and the pandemic ends. Under certain conditions, the naturally occurred herd immunity can only occur if everyone is infected with the virus. Previous study of herd immunity assumes a static population, where individual does not move from place to place in simulation [3-5]. The result may underestimate the severity of COVID-19 pandemic.

\section{Methodology}

This study analyzed 100 freely moved people in a square area using simulation. The time scale of one second in simulation represents one day in real life. Each person continuously moves to a random position in $\mathrm{n}$-seconds time. A new random position is given once the old one is reached. The time duration $\mathrm{n}$ can be adjusted to represent different social activity level (larger $\mathrm{n}$ means less social activity). This semi-random moving pattern is set to represent actual behavior of people in a community. The simulation runs with 0 infected person first to determine the daily risky contact per person. In real life, this means how many times a person may have close contact to others that can potentially transmit disease each day. Once the simulation starts, the patient zero turns infectious (red) from healthy (blue). The virus is transmitting if the healthy person (blue) touches the infectious person (red). The infected person enters the latent period (yellow) of 6 days [6-8], where the person carries virus but not infectious to others. After that, the infected person enters the infectious period (red) of 9 days [9]. After the infectious period, the person gets immunized (purple) and can no longer pass disease to others or get sick again. The simulation continues until no more infectious person exists in the sandbox (Figure 1). At the end of the simulation, the daily personal contact number, " $R$ " value for the first patient, percentage of total infected population, and duration of pandemic are recorded. The "R" value refers to number of people each patient can infect before the end of infectious period. We only recorded the $\mathrm{R}$ value for patient zero as it changes with increasing immunized population. Each simulation is repeated three times and their average value is recorded in Table 1. The total infected population and the pandemic duration is plotted against the daily risky contact per person (Figure 2). 


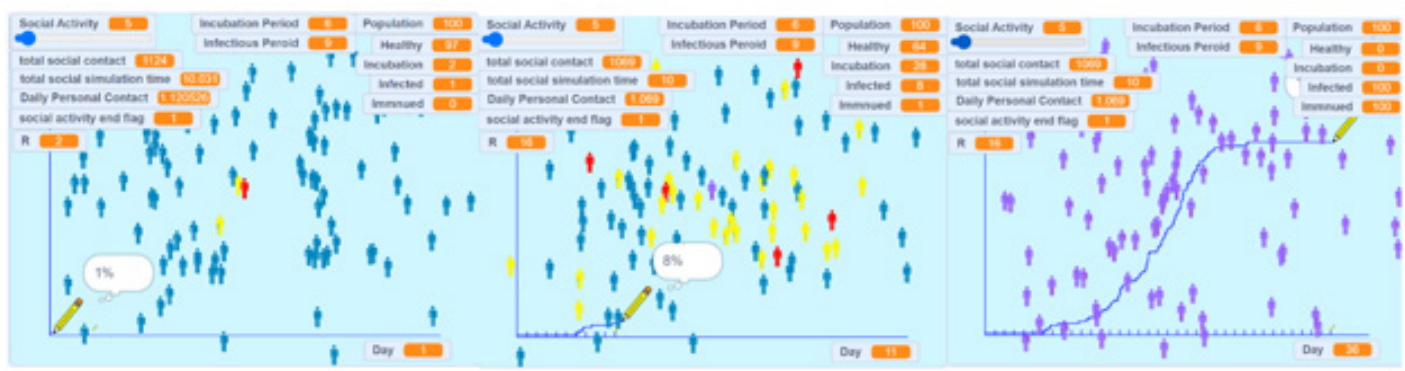

Figure 1: COVID-19 Dynamic Similariton: Day 1 (Left), Day 11 (Center), and Day 36 (Right). Red stands for infectious person, yellow stands for incubation person, blue stands for healthy person and purple stands for immunized person. Entire population (except patient zero) is in a constant semi random motion.
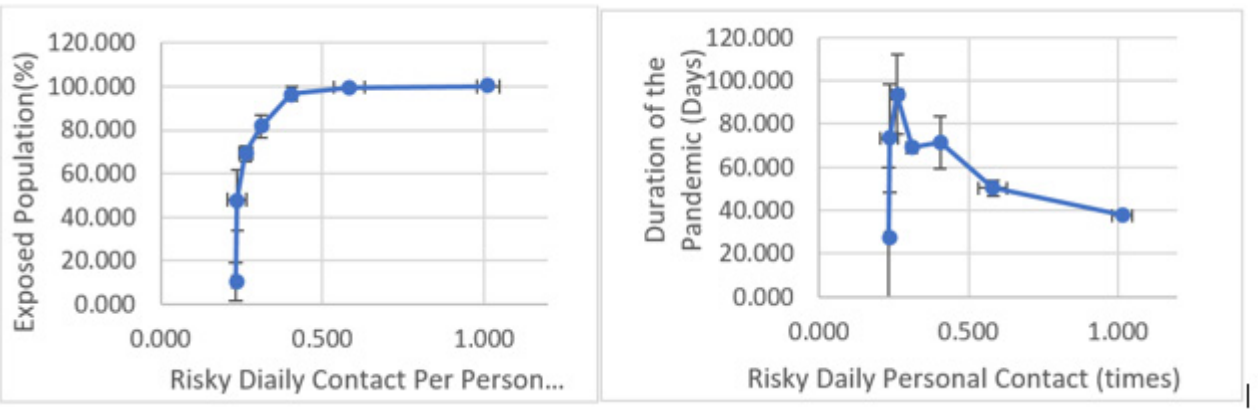

Figure 2: Contact Times Per Person Per Days vs. Final Exposed Population for COVID-19 simulation. The standard deviation (black margin) is calculated from three repeated runs.

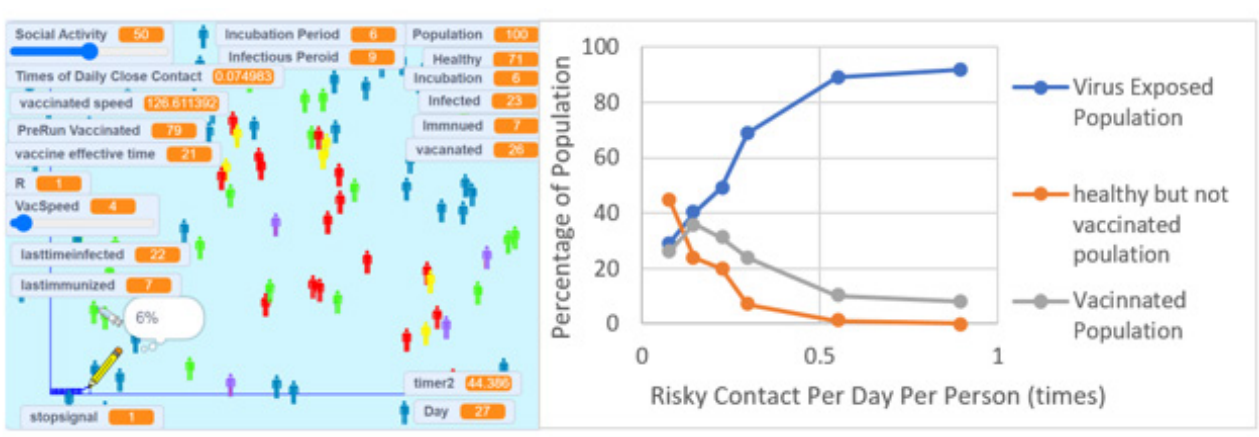

Figure 3: Pandemic in vaccinated Simulation (left) Red means infected, yellow is incubation, purple is naturally immunized. Green is vaccine immunized; blue is healthy but unexposed to the virus. Syringe means the vaccination program that intends to cover the whole population in 120 days. Percentage of Final infected Population with a 120 days vaccinated program.

\section{Result Interpretation and Discussion}

The number of daily risky contact per person is direct related to the total infectious population of the pandemic. A lower daily contact number will allow a larger percentage of the population not infected when pandemic ends. COVID-19 will infect everyone if the average person in society has one times of risky contact per day. If the average person has 0.23 times or less risky contact per day, the virus is more likely to die down without further spreading to other people (Figure 2 Left). The key point for Disease prevention and control measures is to reduce the number of risky contacts among peoples. Mandatory mask-wearing and social distancing makes each contact less risky and thus reduce the number of effective risky contact. Remote learning, working from home and travel restriction will directly reduce the numbers contacts.

The high contagious of SARS-Cov-2 is due to three factors suggested by the simulation:

- The virus has long incubation and infectious period.

- $\quad$ Most contagious person still moves around in society due to asymptomatic infection or mild symptoms.

- The condition of risky contact between two people is easily achieved as the disease is airborne transmitted. 


\section{Vaccinated Result}

The study of a vaccination program is presented for comparison. The program is set to distribute the vaccine to the entire population in 120 days. The distribution of the vaccine starts at the same time of the patient zero get infectious. The vaccine program represented by a syringe freely moves in the sandbox and gives the vaccine to the person in contact (Figure 3). The moving speed is adjusted to reach approximately 120 days of delivery time to the whole population. The result suggests the vaccination program protect part of the population that are not vaccinated only if the daily risky contact per person is below 1 . If the value is above 1 , the vaccine only protects those who are vaccinated. All unvaccinated person will be infected.

Table 1: The simulation result of COVID-19 simulation. Each value is the average of three simulations with identical parameter.

\begin{tabular}{|c|c|c|c|c|}
\hline Social Activity & $\begin{array}{c}\text { Times of Risky Contact Per Day Per } \\
\text { Person }\end{array}$ & Patient Zero R Value & Virus Exposed Population (\%) & Duration of Pandemic (Days) \\
\hline 5 & 1.015 & 14 & 100 & 37.667 \\
\hline 10 & 0.583 & 6.667 & 99.667 & 50.333 \\
\hline 20 & 0.406 & 5.333 & 96.667 & 71.333 \\
\hline 30 & 0.31 & 4.333 & 81.667 & 69 \\
\hline 40 & 0.262 & 1.667 & 69.333 & 93.667 \\
\hline 50 & 0.237 & 1.667 & 48 & 73.333 \\
\hline 60 & 0.233 & 1.333 & 10.333 & 27.667 \\
\hline
\end{tabular}

\section{Acknowledgement}

This work is dedicated to the memorial of Dr. Runtao Yan for his guidance and mentorship. The idea is based on a course project of the first author. Special thanks to MIT scratch program and David Zhang for his teaching and support. Special thanks to Cing-Dao Kan for his valuable suggestions. Source Code of the simulation program is shared at https://scratch.mit.edu/projects/486194245/

\section{Conflict of Interest}

No conflict of interest.

\section{References}

1. Herd Immunity (2021) WebMD.

2. Aschwanden C (2020) The false promise of herd immunity for COVID-19. Nature 587(7832): 26-28.

3. FRED (2021) Web.

4. Herd Immunity (2021) History of Vaccines.
5. Mondal S, Mukherjee S, Bagchi B (2020) Mathematical modeling and cellular automata simulation of infectious disease dynamics: Applications to the understanding of herd immunity. J Chem Phys 2020; 153(11): 114119.

6. Qin J, You C, Lin Q Hu T, Yu S, et al. (2020) Estimation of incubation period distribution of COVID-19 using disease onset forward time: a novel cross-sectional and forward follow-up study. medRxiv.

7. Lauer SA, Grantz KH, Bi Q, Forrest K Jones, Qulu Zheng, et al. (2020) The Incubation Period of Coronavirus Disease 2019 (COVID-19) From Publicly Reported Confirmed Cases: Estimation and Application. Ann Intern Med 172(9): 577-582.

8. Wölfel R, Corman VM, Guggemos W, Michael Seilmaier, Sabine Zange, et al. (2020) Virological assessment of hospitalized patients with COVID-2019. Nature 581(7809): 465-469.

9. Cevik M, Tate M, Lloyd O, Maraolo AE, Schafers J, et al (2021) SARSCoV-2, SARS-CoV, and MERS-CoV viral load dynamics, duration of viral shedding, and infectiousness: a systematic review and meta-analysis. Lancet Microbe 2(1): e13-e22. 